\title{
Pseudoaneurysm of Left Internal Thoracic Artery Graft After Coronary Artery Bypass Grafting: Diagnosis by Transthoracic Echocardiography
}

\author{
Kazuyo Kurozumi*, Chisato Izumi**, Kiyomi Goto*, Sumiyo Hashiwada*, \\ Shuichi Takahashi* and Masahiko Matsumoto*** \\ *Department of Clinical Pathology, \\ **Department of Cardiology, \\ ***Department of Cardiovascular Surgery, Tenri Hospital, Tenri, Japan
}

\begin{abstract}
A cystic lesion between the left internal thoracic artery (LITA) graft and left anterior descending artery (LAD) flow was detected by routine transthoracic echocardiography in a patient after coronary artery bypass grafting. The cystic lesion communicated with the anastomotic site of the LITA graft to LAD, and it was diagnosed as a pseudoaneurysm of the LITA graft by transthoracic echocardiography.

Because pseudoaneurysm of a coronary bypass graft is a potentially fatal complication after coronary artery bypass grafting, it is important to detect the LITA graft flow at the anastomotic site by transthoracic echocardiography in routine examination.

(J Echocardiogr 2004; 2: 80-82)
\end{abstract}

Key words: transthoracic echocardiography, pseudoaneurysm, coronary bypass graft

Pseudoaneurysm of a saphenous vein graft is a rare complication after coronary artery bypass grafting [1-2]. The diagnosis is usually made by angiography and sometimes by computed tomographic scan [3], magnetic resonance imaging [3-4], and transesophageal echocardiography[4]. Recent technological advancements in color Doppler methods have enabled us to evaluate the coronary artery flow by transthoracic echocardiography (TTE) [5]. We report a patient with a pseudoaneurysm of the LITA graft at the anastomotic site which was diagnosed by TTE.

\section{Case}

A 69-year-old woman was referred to our hospital with a recommendation for coronary artery bypass

Received July 29, 2004; revision received August 30, 2004; accepted September 11, 2004

Address for correspondence: Kazuyo Kurozumi Department of Clinical Pathology, Tenri Hospital, 200 Mishima-cho, Tenri-city, Nara 632-8552, Japan. Telephone: +81-743-63-5611

Fax: +81-743-62-5576

E-mail: takaecho@tenriyorozu-hp.or.jp

(C) 2003 Journal of Echocardiography grafting. Preoperative coronary angiography showed triple-vessel coronary artery disease. The left internal thoracic artery (LITA) graft was placed on the distal left anterior descending artery (LAD) and a saphenous vein graft on the left circumflex artery. It was difficult to detect the $\mathrm{LAD}$ during surgery because it ran deep in the epicardial fatty tissue.

Postoperative course was uneventful, and routine TTE (Acuson Sequoia) was performed 11 days after surgery. As the high frequency transducer was positioned at the third intercostal space, the LITA graft and LAD were identified as tubular structures containing color Doppler signals. A cystic lesion with a diameter of $2 \mathrm{~cm}$ was detected between the LITA graft and LAD at the anastomotic site (Figure 1). Mosaic color flow signal was detected from the anastomotic site of the LITA graft to the cystic lesion. The flow signal to the cystic lesion was detected during the systolic phase.

It was diagnosed as a pseudoaneurysm of the LITA graft at the anastomotic site by TTE. Coronary angiography (Figure 2) was performed 14 days after surgery. The right anterior oblique view of angiography showed contrast leakage from the anastomotic site of the LITA graft, confirming the echocardiographic findings. She 


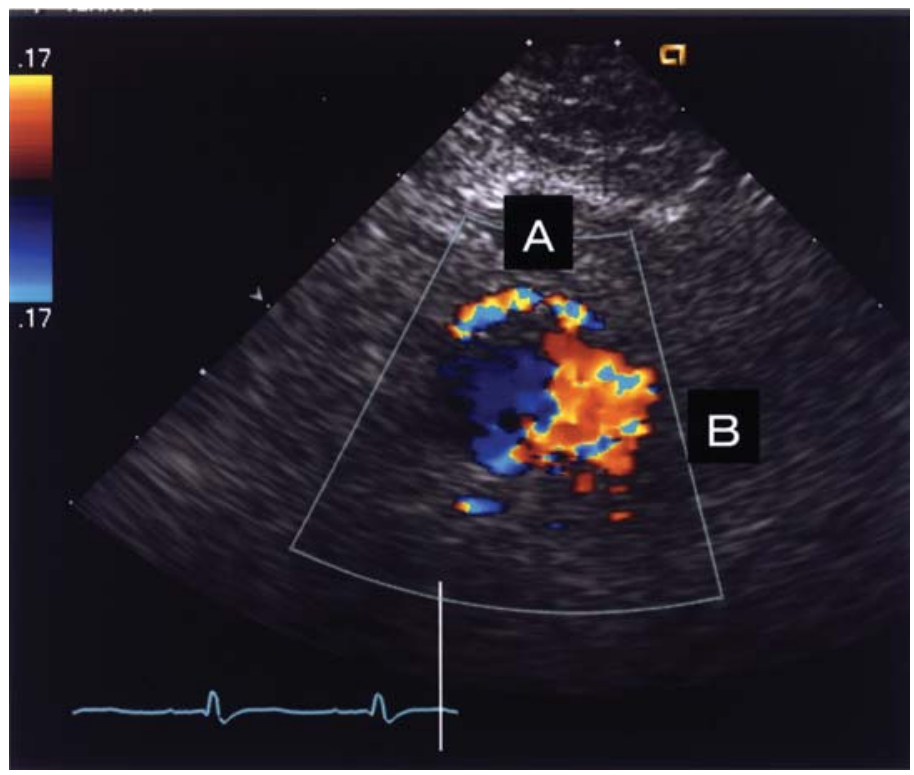

Fig. 1. Mosaic color flow signal was detected from the anastomotic site of the LITA graft (A) to the cystic lesion (B).

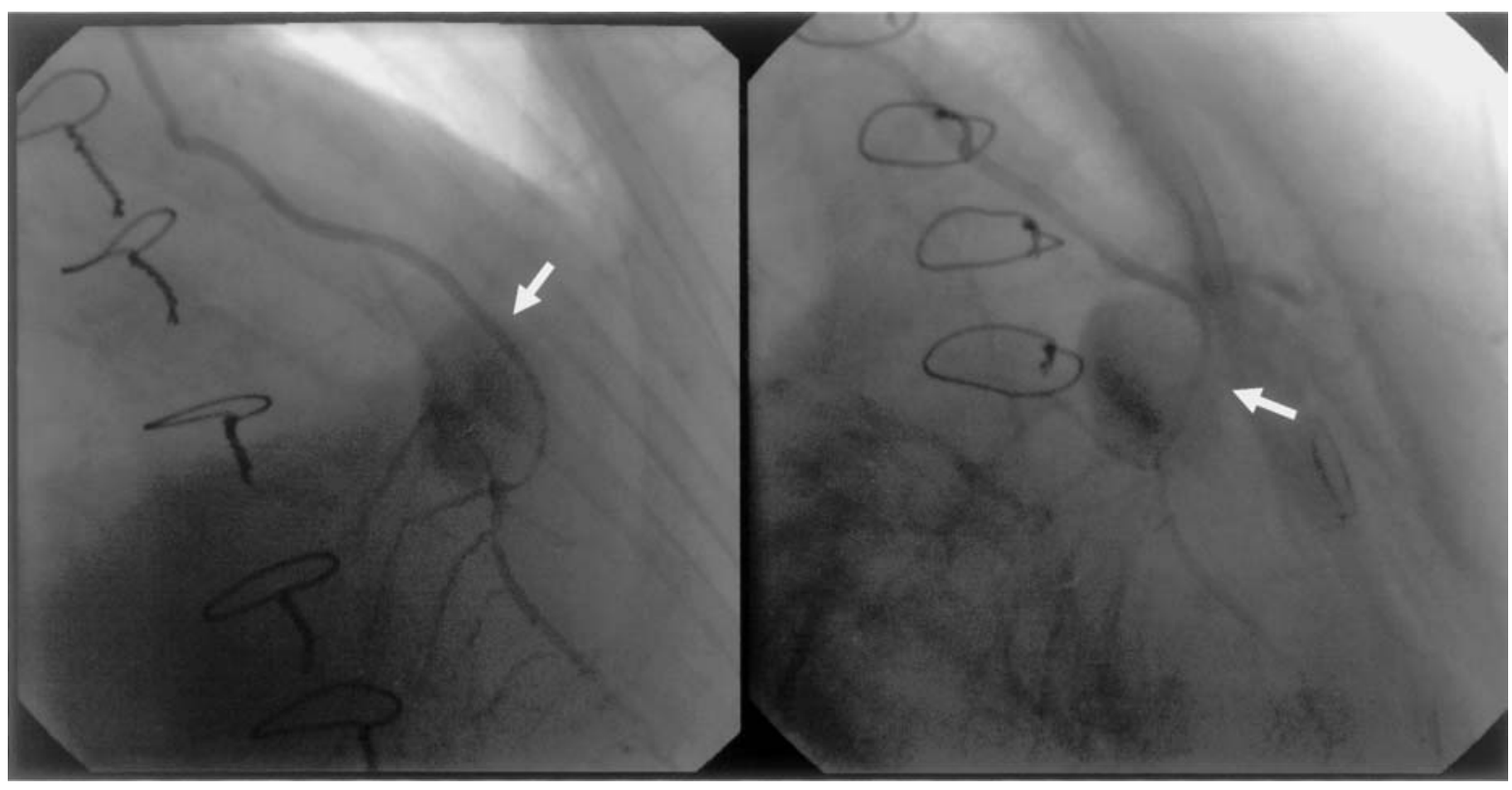

Fig. 2. Right anterior oblique view of angiography showed contrast leakage (arrow) from the anastomotic site of the LITA graft.

thus underwent surgical repair of the pseudoaneurysm of the LITA graft for the purpose of preventing rupture. Intraoperative findings showed that the pseudoaneurysm communicated with the LITA graft at the anastomotic site via a small tear. She was discharged 23 days after surgical repair of the pseudoaneurysm.

A pseudoaneurysm of a saphenous vein graft is usu- ally diagnosed by angiography. Computed tomographic scan [3], magnetic resonance imaging [3-4], and transesophageal echocardiography [4] were also reported to be useful in the diagnosis of pseudoaneurysms. To our knowledge, there were three reports about the pseudoaneurysm of the LITA graft, but it was diagnosed by angiography. Therefore this is 
the first case report of a pseudoaneurysm diagnosed by TTE. Recent technological advancements in color Doppler methods enabled us to identify the LITA graft flow at the anastomotic site by TTE. The LITA graft, LAD flow, and pseudoaneurysm of the LITA graft were detected by routine TTE in our case. Pseudoaneurysm of a saphenous vein graft develops at the distal or proximal anastomotic site in many cases, and rupture [2] and fistula [6] of pseudoaneurysms have been reported. Because pseudoaneurysm of a coronary bypass graft is a potentially fatal complication after coronary artery bypass grafting, it is important to detect the LITA graft flow at the anastomotic site by TTE in routine examination.

\section{References}

1. Haan HPJ, Huysmans HA, Weeda HWH, Bosker HA, Buis B. Anastomotic pseudoaneurysm after Aorto-coronary bypass grafting. Thoracic Cardiovasc Surg 1984; 33: $55-56$.
2. Mohara J, Konishi H, Kato M, Misawa Y, Kamisawa O. Saphenous vein graft pseudoaneurysm rupture after coronary artery bypass grafting. Ann Thorac Surg 1998; 65: 831-832.

3. Steg PG, Benacerraf M, Chatel D, Laissy JP. False aortic aneurysm due to rupture of an aortocoronary saphenous vein bypass graft. Circulation 1997; 96: 3778.

4. Khabeishvili G, Shaburishvili T, Wann S, Sampson C, Manley JC. Saphenous vein graft pseudoaneurysm: Diagnosis by transesophageal echocardiography and magnetic resonance imaging. J Am Soc Echocardiogr 1995; 8: 338-340.

5. Hozumi T, Yoshida K, Ogata Y, et al. Noninvasive assessment of significant left anterior descending coronary artery stenosis by coronary flow velocity reserve with transthoracic color Doppler echocardiography. Circulation 1998; 97: 1557-1562.

6. Dabboussi M, Saade YA, Poncet A, Baehrel B. Fistula between a saphenous vein graft aneurysm and the pulmonary artery trunk. Ann Thorac Surg 2001; 71: 13561358. 